\title{
The Effect of Long-Term TENS on Persistent Neuroplastic Changes in the Human Cerebral Cortex
}

\author{
Raf L.J. Meesen, ${ }^{1,2,3 *}$ Koen Cuypers, ${ }^{1,3}$ John C. Rothwell, ${ }^{4}$ \\ Stephan P. Swinnen, ${ }^{2}$ and Oron Levin ${ }^{2}$ \\ ${ }^{1}$ REVAL-Rehabilitation and Health Care Research Center, Department of Healthcare, \\ University College of Limburg, Hasselt, Belgium \\ ${ }^{2}$ Motor Control Laboratory, Research Center for Movement Control and Neuroplasticity, \\ Department of Biomedical Kinesiology, Group Biomedical Sciences, K.U. Leuven, Belgium \\ ${ }^{3}$ Hasselt University, Biomedical Research Institute, Agoralaan, Diepenbeek 3590, Belgium \\ ${ }^{4}$ Sobell Department of Neurophysiology, Institute of Neurology, Queen Square, \\ London, United Kingdom
}

\begin{abstract}
The long-term effect of daily somatosensory stimulation with transcutaneous electrical nerve stimulation (TENS) on reorganization of the motor cortex was investigated in a group of neurologically intact humans. The scalp representation of the corticospinal projection to the finger (APB, ADM) and forearm (FCR, ECR) muscles was mapped by means of transcranial magnetic stimulation (TMS) before and after a 3-week intervention period, using map area and volume, and topographical overlaps between the cortical motor representations of these muscles as primary dependent measures. Findings revealed a significant increase in cortical motor representation of all four muscles for the TENS group from pre to posttest (all, $P \leq 0.026$ ). No significant changes in cortical motor representations were observed in the control group. The present observations highlight the potential benefit of sensory training by means of TENS as a useful complementary therapy in neurorehabilitation. Hum Brain Mapp 32:872-882, 2011. (C) 2010 Wiley-Liss, Inc.
\end{abstract}

Key words: somatosensory stimulation; corticomotor representations; neuroplasticity; transcranial magnetic stimulation (TMS)

Contract grant sponsor: Flanders Fund for Scientific Research (FWO Project); Contract grant number: G.0483.10N; Contract grant sponsor: Koen Cuypers is sponsored by the Special Research Fund UHasselt.

*Correspondence to: Raf L.J. Meesen, REVAL Research Group, Department of Healthcare, PHL University College, Guffenslaan 39, Hasselt 3500, Belgium. E-mail: raf.meesen@phl.be

Received for publication 25 September 2009; Revised 12 March 2010; Accepted 14 March 2010

DOI: $10.1002 / \mathrm{hbm} .21075$

Published online 9 June 2010 in Wiley Online Library (wileyonlinelibrary.com).

\section{INTRODUCTION}

Somatosensory stimulation activates large parts of the motor and sensory networks, both in the contralateral and ipsilateral hemispheres [Deletis et al., 1992; Duclos et al., 2007; Matteis et al., 2003; Naito et al., 1999; Nelles et al., 1999; Nudo et al., 1996; Radovanovic et al., 2002]. For example, medical imaging techniques have shown activation in contralateral sensorimotor cortex, inferior parietal cortex (bilateral), ipsilateral cerebellum, and (medial) premotor areas [Carel et al., 2000; Naito et al., 1999; Nelles et al., 1999]. This activity reflects the anatomical connectivity of the stimulated M1/S1, known to possess 
direct monosynaptic connections with all of the aforementioned areas [Classen et al., 1998; Nelles et al., 2001].

The effect of somatosensory stimulation on corticomotor excitability has been explored in humans by using transcranial magnetic stimulation (TMS) during and/or after the application of e.g., electrical nerve stimulation [Fraser et al., 2002; Hamdy et al., 1998; Ridding et al., 2001], muscle-tendon vibration [Rosenkranz and Rothwell, 2003; Steyvers et al., 2003a, b], cyclical passive movement [Lewis and Byblow, 2004; Mace et al., 2008], or temporary deafferentation [Ziemann et al., 1996]. These observations have recently been linked with the emergence of lasting facilitation or depression of excitability of neuronal populations in the primary motor cortex [Forner-Cordero et al., 2008; Mace et al., 2008; Rosenkranz and Rothwell, 2006; Rosenkranz et al., 2008; Steyvers et al., 2003a]. Yet, the longlasting changes in corticomotor excitability induced by direct activation of Ia afferent pathways via passive movement or muscle vibration were somewhat more feeble and/or focal [Lewis and Byblow, 2004; Mace et al., 2008] as compared with those scenarios in which facilitation (or depression) in excitability of the corticospinal projections to targeted musculature were monitored following training with electrical nerve stimulation [Fraser et al., 2002; Hamdy et al., 1998; Ridding et al., 2001; Tinazzi et al., 2005].

Electrical nerve stimulation in general, and transcutaneous electrical nerve stimulation (TENS) in particular have been applied successfully in neurorehabilitation, such as in the treatment of stroke [Levin and Hui-Chan, 1992; Ng and Hui-Chan, 2007; Sonde et al., 1998], urinary symptoms [Skeil and Thorpe, 2001], spinal cord injury [Fung and Barbeau, 1994; Goulet et al., 1996], multiple sclerosis [Armutlu et al., 2003; Miller et al., 2007], writer's cramp [Tinazzi et al., 2006] and/or to reduce movement disorders caused by tremor, myoclonia, or dystonia [Bending and Cleeves, 1990; Foley-Nolan et al., 1990; Toglia and Izzo, 1985]. This body of literature suggests that daily training with TENS may effectively activate larger parts of the underlying distributed sensorimotor networks of the brain. Studies that have evaluated the long-term effects of TENS on the reorganization of corticomotor representations are sparse. Nonetheless, a recent study revealed that multiple sessions of peripheral nerve stimulation can facilitate training effects on motor function after subacute stroke [Conforto et al., 2010].

The present study aimed to reveal the effects of a daily somatosensory stimulation with TENS over the right abductor pollicis brevis (APB) muscle on cortical representations of hand and forearm muscles in healthy volunteers (see Fig. 1). On the basis of the known connectivity between primary sensory and motor cortical areas [Quartarone et al., 2003; Zarzecki et al., 1978], we hypothesized positive effects of the sensory intervention on the motor map representation(s) after 3 weeks of daily training. More specifically, as a result of the existence of (1) widespread connectivity between, e.g., the median and ulnar

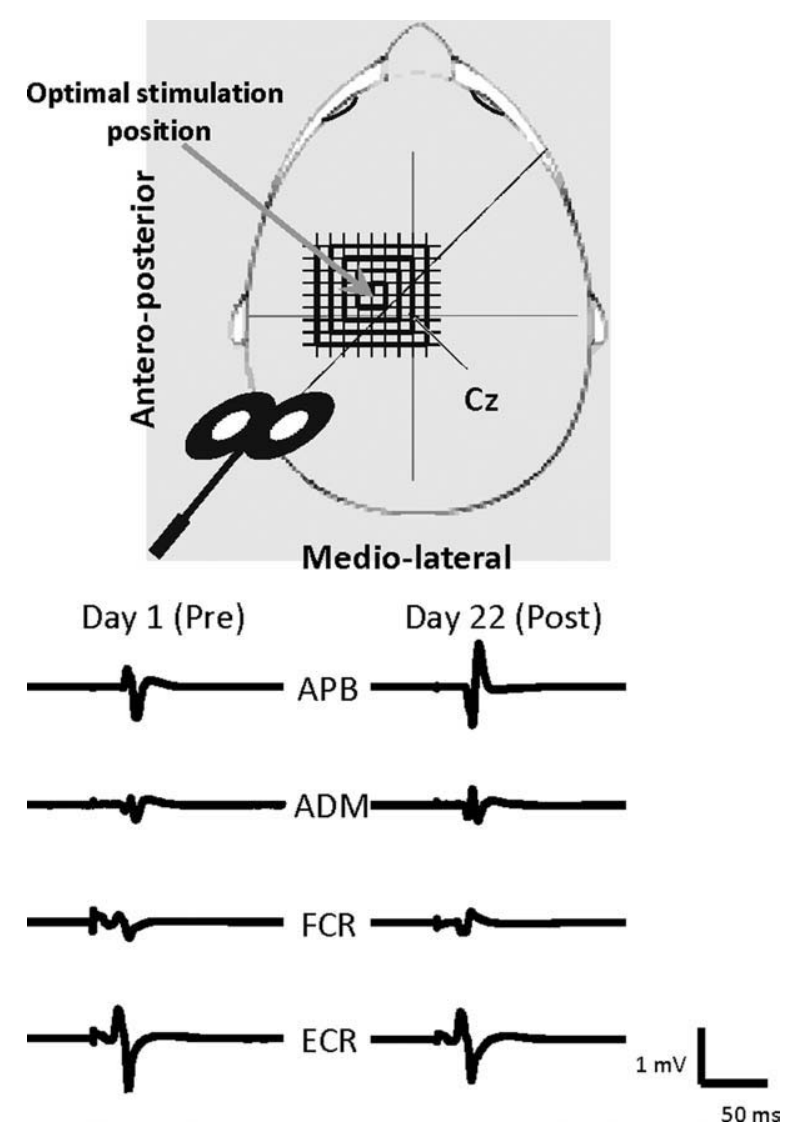

Figure I.

Illustration of the TMS mapping procedure.

nerves [Kimura et al., 1983] and/or (2) simultaneous activation of primary and secondary afferent networks [Quartarone et al., 2006], we hypothesized that TENS applied to the APB would induce a global effect on hand (APB, ADM) and forearm (FCR, ECR) representations. Changes in motor cortex representations were assessed by means of a TMS mapping protocol, examining differences in loci and size of the cortical motor maps of the hand and forearm musculature [Thickbroom et al., 1999; Wassermann et al., 1992; Wilson et al., 1996]. This technique has been used previously to assess changes in corticomotor representations of upper/lower limb musculature following limb amputation [Cohen et al., 1991; Fuhr et al., 1992] or specific skill learning [PascualLeone et al., 1993, 1994].

\section{MATERIALS AND METHODS}

\section{Subjects}

Participants were 24 healthy and neurologically intact right-handed volunteers (10 males, 14 females, mean age 27.4, SD 14.8, range 18-54 years). They were naive about 
the purpose of the experiment, were screened for potential risk of adverse events during TMS [Wassermann, 1998], and provided written informed consent prior to participation. Handedness was determined by the Edinburgh Handedness Inventory [Oldfield, 1971]. The experimental procedures were approved by the local Ethics Committee for Biomedical Research at Katholieke Universiteit Leuven, according to the Declaration of Helsinki.

\section{Experimental Procedures}

Participants were randomly assigned to two groups ( $n=$ 12 each): TENS and control (CONTROL). All subjects underwent two TMS mapping sessions, i.e., prior to the start of (at day 1) and following the intervention (at day 22). The intervention consisted of daily sessions over a period of 21 days. Participants in the control group preserved their normal daily activities without any intervention.

TENS (biphasic symmetrical rectangular pulse-wave at $100 \mathrm{~Hz}, 250 \mu$ s pulse width) was delivered for a total duration of 60 min per day to the right APB muscle, using a constant current stimulator (Intelect Digitens, Chattanooga Group, Hixson, TN). Self-adhesive electrodes (dura Stick II, $1.5 \mathrm{~cm} \times 4 \mathrm{~cm}$ ) were placed on the belly of the right $\mathrm{APB}$ and their locations were marked over the skin with a surgical pen to preserve locations across the daily sessions. Stimulus intensity was set at a sensory threshold just below motor threshold, producing a continuous tingling sensation in the stimulated area without any visible muscle twitch or pain.

\section{Transcranial Magnetic Stimulation}

TMS mapping of the right $\mathrm{APB}$, abductor digiti minimi (ADM), flexor carpi radialis (FCR), and extensor carpi radialis longus (ECR) was done by means of a magnetic stimulator (Dantec MagLite r-25, Medtronic, Skovlunde, Denmark). Single-pulse magnetic stimuli were delivered with a figure-of-eight coil (MC-B70 magnetic coil transducer; outer radius diameter: $50 \mathrm{~mm}$; maximal output: 1.5 Tesla). For each subject a customized cap was made out of thermoplastic material (Aquaplast-T Solid, $20 \mathrm{~cm} \times 15 \mathrm{~cm}$ $\times 0.24 \mathrm{~cm}$, Sammons Preston Polyon, Cedarburg) with references to anatomical landmarks (left and right external auditory meatus and occiput) to ensure reproducibility of measurements across the experiment. An orthogonal coordinate system referenced to the vertex was marked on each cap. The coil was positioned tangentially to the scalp over the subjects' left hemisphere with the coil handle pointing backward and rotated $45^{\circ}$ away from the midsagital line (see Fig. 2).

The optimal location (hotspot) for eliciting motor evoked potentials (MEPs) from the right $\mathrm{APB}$, i.e., the target muscle, was identified and marked with a soft-tip pen to ensure reproducibility of coil positioning in each participant. In all cases, a response of the ADM, FCR, and ECR was also evoked in this position. Next, the rest motor threshold (rMT) was determined at the optimal scalp positions of the APB, ADM, FCR, and ECR. For all muscles, the rMT was defined as the lowest intensity of magnetic stimulation required to evoke MEPs larger than $50 \mu \mathrm{V}$ amplitude in at least 5 of 10 trials in the relaxed muscle. The stimulation intensity for mapping was then set at $120 \%$ of the APB rMT. This intensity was preserved during both the pre and postmapping sessions. A grid of $225(15 \times 15)$ positions, spaced $1 \mathrm{~cm}$ along both the medio-lateral and antero-posterior axes, was marked on each cap. In producing maps, single TMS pulses were applied in $1 \mathrm{~cm}$-steps in a clockwise spiral course, beginning at the hotspot of the APB muscle (see Fig. 1). Each position was stimulated 8 times (interstimulus interval: 5-8 s, at random) before moving to the adjacent grid point, until the border of the motor maps of all four muscles was defined.

\section{EMG Recordings}

Electromyographic (EMG) signals from the APB, ADM, FCR, and ECR of the right upper limb were collected by means of disposable, self-adhesive disc electrodes (Nutrode, $\mathrm{Ag}-\mathrm{AgCl}$ sensor with Hydrogel, $35 \mathrm{~mm}$ diameter, GE Medical Systems Accessories Europe, Nanterre Cedex, France). For each subject, specific electrode placement for each of the four muscles was photographed and saved in a database. Skin surface was carefully shaved and degreased prior to electrode placement to achieve optimal conduction. The electrodes were additionally fixed to the skin with tape to ensure maximal contact (Leukopor, skin-friendly, nonwoven tape, 1.25 $\mathrm{cm}$, BSN Medical GmbH and Co, Hamburg, Germany). After amplification (gain $=1,000$ ) and bandpass filtering (4-1,500 Hz) (MEGA MESPEC 8000, Finland), the recorded EMG signals were digitized at 5,000 $\mathrm{Hz}$ (CED Signal Version 3.03, Cambridge Electronic Design, Cambridge, UK) and were stored on a laboratory computer for offline analysis. Data collection was initiated $50 \mathrm{~ms}$ prior to the delivery of TMS and lasted $150 \mathrm{~ms}$. EMG activity from all four muscles was continuously monitored online during the mapping session. Background EMG activity was minimized by continuous online EMG monitoring during the experiment, as well as by offline analysis of the trials.

\section{Data Analysis}

The size of the APB, ADM, FCR, and ECR MEPs were measured offline by calculating the peak-to-peak amplitude of each waveform in a time window from 10 to 40 $\mathrm{ms}$ after the TMS stimulation pulse onset. The number of active positions in each map was determined as points whose stimulation evoked a mean MEP in the target muscles with a peak-to-peak amplitude of at least $100 \mu \mathrm{V}$. Three-dimension representations of mean motor outputs 
(a)

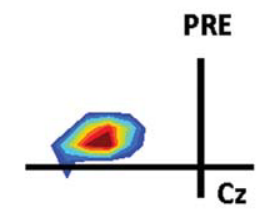

$2 \mathrm{~cm}$
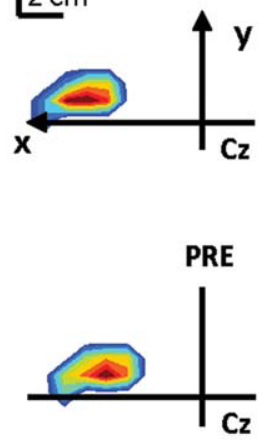

$2 \mathrm{~cm}$

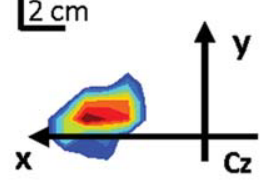

TENS Group

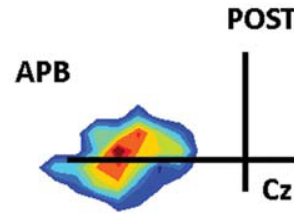

ADM

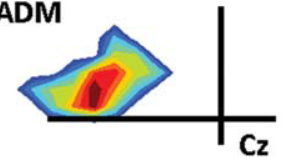

POST

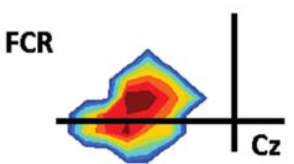

ECR

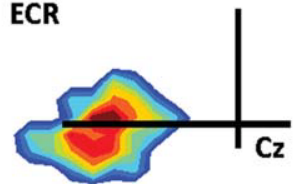

(b)

\section{Control Group}
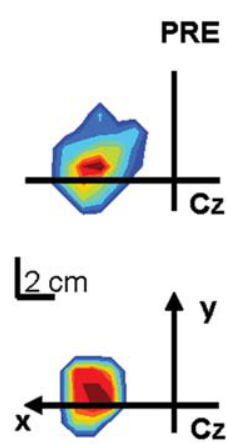

ADM

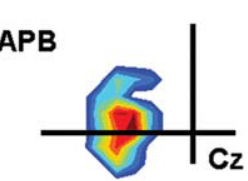

$\%$ max MEP

90-100

80-90

70-80

70-80

$60-70$
$50-60$

$50-60$
$40-50$

$30-40$

30-40

20-30

$10-20$
$0-10$

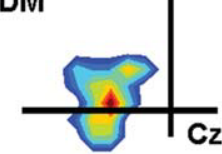

PRE
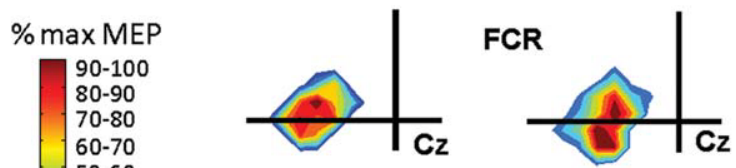

POST

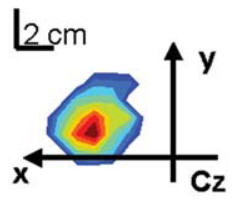

$\% \max$ MEP

90-100

80-90

$70-80$

60-70

50-60

40-50

ECR

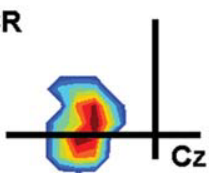

Figure 2.

Representative map areas of the ABP, ADM, FCR, and ECR muscles before (Pre-left hand column) and after 3 weeks (Post - right hand column) of sensory intervention with TENS (a) as compared to control (b). [Color figure can be viewed in the online issue, which is available at wileyonlinelibrary.com.]

for the four muscles were then composed by linear interpolation of the mean MEP amplitudes between adjacent stimulation positions (Matlab 6.5, MathWorks, Inc.).

For both pre and postmapping sessions, the cortical motor representation of the APB, ADM, FCR, and ECR was defined as the number of stimulus positions whose stimulation evoked a mean MEP in the target muscle with a peak-to-peak amplitude of at least $100 \mu \mathrm{V}$ (= "active" stimulation positions). The peak-to-peak amplitudes obtained at the same stimulation site were averaged and normalized by their respective mean MEP amplitudes at the hotspot. The motor representation area of each muscle was defined as the number of stimulus positions whose stimulation evoked a mean MEP in the target muscle with a magnitude of at least $10 \%$ of its respective normalized peak. Map area referred to the contour, whereas Map volume referred to the sum of the mean amplitudes at all active stimulation positions. The center of gravity (CoG) was computed separately for each muscle as a measure of the amplitude-weighted centre of the motor representational map [Wassermann et al., 1992]. It is expressed as a bivariate measurement with a mediolateral $(x)$ and anteroposterior coordinate $(y)$, using the following formula: CoG $=\left[\sum a_{i} x_{i} / \sum a_{i}, \sum a_{i} y_{i} / \sum a_{i}\right]$, for stimulation position coordi- nates $x_{i}, y_{i}$ and amplitudes $a_{i}$. The magnitude of the CoG displacement vector, i.e., the Euclidean distance between the CoG locations at day 1 (preintervention mapping session) and day 22 (postintervention mapping session) was calculated.

\section{Statistics}

Advanced linear models applications (STATISTICA 6.0, StatSoft Inc.) were used for statistical analysis. Effects of interventions on corticospinal excitability and corticomotor representation were studied using the following dependent measures: mean MEP amplitude at optimal stimulation position (hotspot), location of hotspot, mean map area and mean map volume, map overlaps and the displacement of the map CoG. A $2 \times 2 \times 4$ (GROUP $\times$ TIME $\times$ MUSCLE) repeated measures analysis of variance (ANOVA) was used with GROUP (TENS, CONTROL) as between-subjects factor, and TIME (PRE-POST mapping session) and MUSCLE (APB, ADM, FCR, ECR) as withinsubjects factors. Differences in topographical overlaps between cortical representations of the target (APB) and nontarget (ADM, FCR, and ECR) muscles in the pre and postmapping sessions were tested using a $2 \times 2 \times 6$ 
TABLE I. Mean ( \pm SD) magnitudes of the MEPs $(m V)$ in the pre and postintervention mapping sessions for the TENS and Control groups

\begin{tabular}{lccccc}
\hline & \multicolumn{2}{c}{ TENS } & & \multicolumn{2}{c}{ Control } \\
\cline { 2 - 3 } \cline { 5 - 6 } & Pre & Post & & Pre & Post \\
\hline APB & $1.81 \pm 0.39$ & $1.83 \pm 0.40$ & & $2.10 \pm 0.39$ & $2.14 \pm 0.40$ \\
ADM & $1.13 \pm 0.24$ & $1.51 \pm 0.30$ & & $0.96 \pm 0.24$ & $0.71 \pm 0.30$ \\
FCR & $0.73 \pm 0.10$ & $0.69 \pm 0.12$ & & $0.39 \pm 0.10$ & $0.40 \pm 0.12$ \\
ECR & $1.26 \pm 0.25$ & $1.15 \pm 0.23$ & & $0.74 \pm 0.25$ & $0.60 \pm 0.23$ \\
\hline
\end{tabular}

ANOVA with the factors GROUP (two levels), TIME (two levels) and MAP OVERLAP (six levels: APB-ADM, APBFCR, APB-ECR, ADM-FCR, ADM-ECR, and FCR-ECR). T-tests were used to estimate whether potential changes in map area, volume or map overlaps differed significantly from zero. The magnitude of displacement of the estimated CoG positions along the anteroposterior and mediolateral axes were statistically compared using a $2 \times 4$ $($ GROUP $\times$ MUSCLE) ANOVA, to determine CoG stability between pre and postmapping sessions. The level of significance was set at $P<0.05$.

\section{RESULTS}

\section{MEP Amplitude at Hotspot}

Three weeks of TENS did not affect the size of MEPs at the hotspots, suggesting that levels of corticospinal excitability at hotspot remained unchanged (Table I). Specifically, the $2 \times 2 \times 4$ (GROUP $\times$ TIME $\times$ MUSCLE) ANOVA revealed a significant main effect for MUSCLE ( $P$ $<0.01$ ) but the remaining main effects and the two and three factor interactions were not significant (all: $P>0.2$ ).

\section{Location of the Hotspot}

The location of the hotspots did not change significantly between pre and postmapping sessions (all muscles: $P>0.08)$.

\section{Map Area and Map Volume}

Examples of individual maps are illustrated in Figure 2a (TENS) and $2 \mathrm{~b}$ (CONTROL). Changes in mean map area and volume of the representations of the target (APB) and nontarget muscles (i.e., ADM, FCR, and ECR) from pre to posttest are demonstrated in Figure 3. Overall, changes in the group mean areas (Fig. 3a) and volumes (Fig. 3b) between pre and postmapping sessions were observed for all four muscles in the TENS group. The control group did not reveal any changes. The aforementioned observations were largely confirmed by the $2 \times 2 \times 4$ (GROUP $\times$ TIME $\times$ MUSCLE) ANOVA, as discussed next.

With respect to map area, the main effects for TIME $(P$ $<0.01)$ and GROUP $(P<0.05)$ and the GROUP $\times$ TIME interaction $(P<0.01)$ were significant. The main effect for MUSCLE $(P=0.079)$ and the remaining two and three factor interactions (all: $P>0.5$ ) were not significant, suggesting that all four muscles present a similar increase in the representation of their map areas after the intervention. This finding enabled us to explore the significant GROUP $\times$ TIME interaction in detail. Specifically, pairwise post hoc tests (Tukey HSD) revealed a significant increase of map area size in POST $\left(M=25.0 \mathrm{~cm}^{2}\right)$ relative to PRE $\left(M=14.9 \mathrm{~cm}^{2}\right)$ mapping session in the TENS group $(P=0.0003)$ while map area sizes in the CONTROL group were statistically identical: $M=14.5$ $\mathrm{cm}^{2}$ (PRE) vs. $M=15.2 \mathrm{~cm}^{2}$ (POST) $(P>0.9)$. Across group effects were also significant: TENS-POST vs. CONTROL-PRE/POST (both, $P<0.005$ ). Finally, $t$-tests for single means (TENS group: POST-PRE comparison to zero) showed that the map area of the target (APB) and the three nontarget muscles (ADM, FCR, and ECR) was significantly increased: APB (69 $\pm 15 \%$ increase POST vs. PRE, $P=0.001)$, FCR $(73 \pm 21 \%$ increase, $P=0.007)$, ECR $(89 \pm 27 \%$ increase, $P=0.008)$, and ADM (54 $\pm 21 \%, P=0.026)$. As expected, no consistent change in map areas between pre and postmapping sessions were observed in the CONTROL group (all muscles, $P>$ $0.3)$.
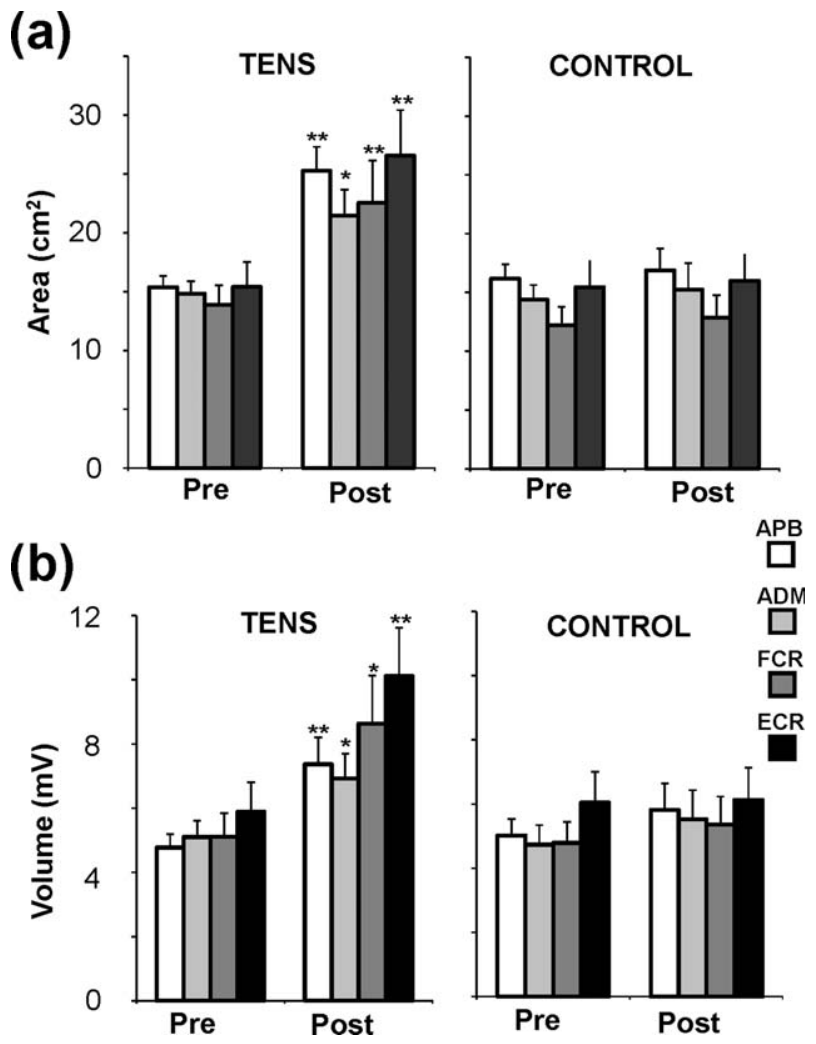

Figure 3.

Group data showing motor representation area and volume of the four muscles at pre and postmapping sessions; $* P<0.05$ and $* * P<0.01$ compared to the Prevalues. 


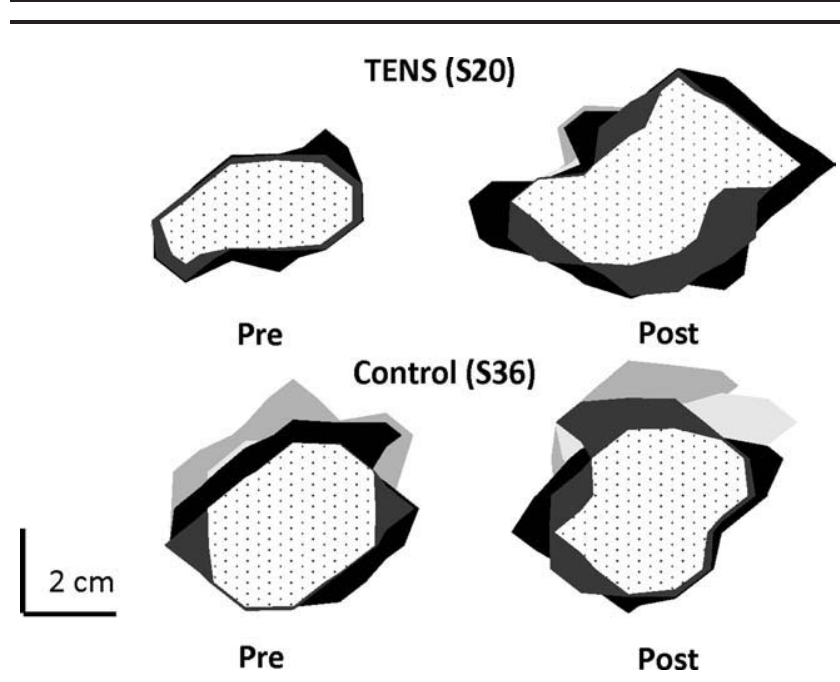

Figure 4.

Representative map areas of the ABP (light-gray), ADM (intermediate gray), FCR (dark gray) and ECR (black) and their overlaps (dotted area) before (Pre-left hand column) and after 3 weeks (Post-right hand column) of sensory intervention with TENS versus control.

With respect to map volume, effects were largely similar but the main effect for GROUP $(P>0.1)$ was not significant. Again, $t$-tests for single means showed that the map volume in the TENS group was significantly increased in all four muscles: $\mathrm{APB}(55 \pm 12 \%, P=0.001), \mathrm{ADM}(46 \pm 18 \%, P=0.026), \mathrm{FCR}$ $(91 \pm 29 \%, P=0.010)$, and ECR $(97 \pm 26 \%, P=0.004)$.

In summary, participants in the TENS group showed a markedly increased map area and volume in all four muscles

\section{Overlaps Between Muscle Cortical Representations}

Representative examples of topographical overlaps between cortical representations of the target (APB) and nontarget (ADM, FCR, and ECR) muscles in the pre and postmapping sessions are illustrated for two individuals (TENS and CONTROL) in Figure 4 . The $2 \times 2 \times 6$ ANOVA revealed significant main effects for TIME $(P<$ $0.01)$ and GROUP $(P<0.05)$ and a significant GROUP $\times$ TIME interaction $(P<0.01)$. The main effect for MAP OVERLAP $(P>0.1)$ and the remaining two and three-factor interactions were not significant (all: $P>0.3$ ). Pairwise post hoc test (Tukey HSD) on the significant GROUP $\times$ TIME interaction revealed a significant increase of map overlap size in the POST $\left(M=19.5 \mathrm{~cm}^{2}\right)$ relative to PRE $\left(M=12.6 \mathrm{~cm}^{2}\right)$ mapping session in the TENS group $(P=$ $0.0008)$ while no enlargements in map overlaps were noticed for the CONTROL group: $M=11.3 \mathrm{~cm}^{2}$ (PRE) vs. $M=11.7 \mathrm{~cm}^{2}$ (POST) $(P>0.9)$. Across group effects were also significant: TENS-POST vs. CONTROL-PRE/POST (both, $P<0.01$ ). Yet, further inspection of our data showed that changes in topographical overlaps of the hand (i.e., $\mathrm{APB}$ and $\mathrm{ADM}$ ) and forearm (i.e., FCR and ECR) musculature after 3 weeks intervention with TENS were more pronounced for some muscles than others. Specifically, significant enlargements in map overlaps were noticed for the representations of the APB, ADM, and ECR (TENS Group, contrast analyses for TIME (Tukey HSD); all, $P \leq 0.034$ ) but not for the aforementioned three muscles with FCR (all, $P>0.06)$. Group means $( \pm \mathrm{SD})$ are shown in Table II.

Further, $t$-tests for single means (TENS group: POSTPRE comparison to 0) indicated that TENS intervention significantly increased the overlaps among cortical maps in the post as compared with the premapping session between the representations of the APB and ADM (PRE vs. POST, $52 \%$ increase; $P=0.024$ ), APB and ECR (PRE vs. POST, $70 \%$ increase; $P=0.002)$ and ADM and ECR (PRE vs. POST, $73 \%$ increase; $P=0.015$ ). Changes in size of overlaps between the FCR and the two finger muscles or FCR and ECR were either marginally significant or did not reach significance (all, $P>0.05$ ). An increase in the size of the topographical overlap of the four cortical maps was also visible and reached marginal significance $(P=$ 0.052).

TABLE II. The effect of training with TENS on overlaps between cortical representations of the target muscle (APB) and the three nontarget muscles (ADM, FCR, and ECR)

\begin{tabular}{|c|c|c|c|}
\hline Cortical representations & Innervation & Pre $\left(\mathrm{cm}^{2}\right)$ & Post $\left(\mathrm{cm}^{2}\right)$ \\
\hline $\mathrm{APB} \cap \mathrm{ADM}$ & median/deep branch of ulnar (APB) and ulnar (ADM) & $12.0(1.9)$ & $18.3(4.8)^{*}$ \\
\hline $\mathrm{APB} \cap \mathrm{FCR}$ & median (APB and FCR) and deep branch of the ulnar (APB) & $11.7(4.2)$ & $17.5(8.7)^{\mathrm{NS}}$ \\
\hline $\mathrm{APB} \cap \mathrm{ECR}$ & median/deep branch of ulnar (APB) and radial (ECR) & $13.0(3.8)$ & $22.0(8.0)^{* *}$ \\
\hline $\mathrm{ADM} \cap \mathrm{FCR}$ & ulnar $(\mathrm{ADM})$ and median $(\mathrm{FCR})$ & $11.8(4.1)$ & $17.1(8.6)^{\mathrm{NS}}$ \\
\hline $\mathrm{ADM} \cap \mathrm{ECR}$ & ulnar (ADM) and radial (FCR) & $13.1(3.7)$ & $22.6(11.2)^{* *}$ \\
\hline FCR $\cap$ ECR & median (FCR) and radial (ECR) & $13.9(3.7)$ & $19.3(11.2)^{\mathrm{NS}}$ \\
\hline $\mathrm{APB} \cap \mathrm{ADM} \cap \mathrm{FCR} \cap \mathrm{ECR}$ & median (APB and FCR), ulnar (APB and $\mathrm{ADM}$ ) and radial (ECR) & $9.8(3.6)$ & $12.8(7.8)^{\mathrm{NS}}$ \\
\hline
\end{tabular}

Data are expressed as group mean \pm standard deviation (SD) for pre (baseline) and postintervention mapping sessions.

NS, not significant.

${ }^{*} P<0.05$ and

${ }^{* *} P<0.01$, Post compared to Prevalues. 
In summary, in addition to a noticeably increased map area in all four muscles, the TENS intervention was also shown to increase the topographical overlaps between cortical representations of the finger and forearm muscles. However, this effect was more prominent for the APB, ADM, and ECR than FCR.

\section{Displacement of the Center of Gravity}

The CoGs of the motor maps did not change between pre and postmapping sessions (all muscles: $P>0.1$ ).

\section{DISCUSSION}

In this study, we investigated the effect of a somatosensory stimulation intervention with TENS across a 3 week period on the cortical motor representations in the intact human brain. Our observations demonstrate for the first time that the size of motor map representations of $\mathrm{APB}$, ADM, FCR, and ECR muscles increased after TENS applied to the median nerve territory over the APB muscle.

The absolute size and spatial location of the map representation depend on factors such as coil orientation [Wilson et al., 1993, 1996], current spread [Roth et al., 1991], coil distance [Thickbroom et al., 1998], and the excitability of the underlying corticospinal projection [Ridding and Rothwell, 1997]. All these parameters were held constant in the present study. Even so, all muscles underwent a considerable increase $(>50 \%)$ in the area of their cortical motor representation in the absence of changes in excitability of the most excitable zone ("hotspot") or the CoG of the motor maps. This suggests that TENS led to a change in the distribution of excitability in the motor output zones to each muscle, with the largest effects in the least excitable peripheral areas. This led to marked changes in the topographical overlaps between cortical representations of the target (APB) muscle and two of the three remaining muscles (i.e., ADM and ECR).

\section{Modulation of Cortical Motor Representation}

Our data, showing enlargement of the borders of cortical maps of the hand and forearm muscles, are in agreement with the findings of previous studies regarding the immediate poststimulation effects with electrical nerve stimulation [Hamdy et al., 1998; Ridding and Rothwell, 1997; Ridding et al., 2001] or muscle vibration [Forner-Cordero et al., 2008], as well as with the long-term effects induced by specific skill learning [Pascual-Leone et al., 1995] or constraint-induced movement therapy in stroke patients [Liepert et al., 2000]. Such changes are usually considered to reflect an expansion of the cortical motor representation of the muscles being investigated [Pascual-Leone et al., 1993, 1995; Ridding et al., 2001; Thickbroom et al., 1999]; for review see [Siebner and Rothwell, 2003]. However, increased motor map expansion does not necessarily signify a true expansion of the underlying cortical projection zone [Ridding et al., 2001]. For example, it has been demonstrated that an increase in the excitability of corticospinal tracts at the original projection would equally lead to an increase in motor map area (e.g., Ridding and Rothwell, 1997]. Nevertheless, further inspection of our data qualifies this possibility. First, the primary increase of the cortical motor map representations of the APB, ADM, FCR, and $\mathrm{ECR}$ in the postintervention mapping session was not accompanied by underlying changes in the excitability of corticospinal projections to those muscles. Specifically, we found neither significant enlargements nor reductions in the amplitudes of MEP at the hotspot between the pre and postmapping sessions. This finding contrasts with the increases in excitability of cortico-cortical and corticospinal circuitry, such as seen after electrical nerve stimulation [Fraser et al., 2002; Hamdy et al., 1998; Ridding and Rothwell, 1997]. However, these rarely last more than $2 \mathrm{~h}$ after the end of the intervention and often show a slight depression $24 \mathrm{~h}$ postintervention [Ridding et al., 2001]. Second, we observed no enlargement of the hotspots, neither in the target $(\mathrm{APB})$ muscle nor in the remaining muscles tested. Specifically, we found no significant changes in the motor representation areas of all muscles at those active sites where the mean amplitude of MEPs, recorded at the pre/postmapping sessions, was larger than $40 \%$ of the mean MEP recorded at the hotspots. In other words, enlargement of cortical motor representations following 3 weeks of TENS occurred around the borders of the cortical motor maps, as established at the premapping session.

The mechanism responsible for the expansion of the maps cannot be determined from the present data. It could be caused by true reorganization of the connectivity patterns in the cortex; alternatively, it could simply result from an increase in the excitability of connections that were already present but not detected by the TMS method. However, the fact that the maps expanded in muscles distant from the site of stimulation is interesting. In this respect, it is plausible that the observed expansion of the output zones to all muscles may have been partially due to high overlaps in topographic and/or neuronal representations between the APB and the remaining muscles.

Interestingly, the observed increase in TENS-induced representational reorganization of cortical maps is neither restricted to the motor areas nor to common innervation pathways (Table II). For example, prolonged (3 weeks) TENS treatment over the median nerve in MS patients has revealed that increases in tactile sensitivity were not restricted to thumb and index finger areas but also expanded to the fifth finger area [Cuypers et al., in press]. The fact that TENS-induced enlargements in the hand motor and/or sensory representations occur at multiple sites, suggests that somatosensory stimulation of peripheral afferent pathways with TENS might spread to nonstimulated parts of the somatosensory network. Electrophysiological and/or neuroimaging mapping 
suggests that this phenomenon may occur both at the peripheral and supraspinal (cortical) levels [Kimura et al., 1983; Krause et al., 2001; Kurth et al., 2000; Sato et al., 2005].

The present findings provide indirect support for a strong connectivity between the somatosensory and motor areas. Electrophysiological animal research has revealed topographically and functionally specific corticocortical excitatory connections between somatosensory areas and primary motor cortex (M1) [Caria et al., 1997; Ghosh and Porter, 1988; Huerta and Pons, 1990; Lucier et al., 1975; Murphy et al., 1975].

\section{The Mechanisms Underlying Motor Reorganization}

We can only speculate on the mechanisms involved in the changes we observed here. Long-lasting changes in cortical motor excitability produced by repetitive central or peripheral stimulation have been shown to depend on long-term potentiation (LTP) or long-term depression (LTD). For example, it has been demonstrated that layer II/III horizontal connections in rat primary motor cortex, which are capable of LTP and LTD, are strengthened during acquisition of a new motor skill [Rioult-Pedotti et al., 2007]. Furthermore, animal studies suggest that LTP-like processes in motor cortex transiently increase synaptic strength, by insertion of glutamate receptors to existing synapses [Harms et al., 2008; Rioult-Pedotti et al., 2007]. In humans, learning of new motor skills is also shown to be associated with LTP-like changes in activity of cortical synapses which are most likely mediated by down-regulation of GABAergic inhibition [Rosenkranz et al., 2007]. Our observations indicated that (1) the 3 weeks intervention with TENS expanded the overlaps between output zones of neighboring muscles without inducing discernible changes in the level of corticospinal excitability around the hotspots, and that (2) enlargement of cortical motor maps occurred around the borders of their preintervention regions. In line with the aforementioned observations, it is reasonable to assume that long stimulation periods may result in the formation of new connections. Studies using pharmacological interventions may help to further unravel these mechanisms.

It is also meaningful to address how the present data can be reconciled with previous reports on the effect of single sessions of TENS on cortical excitability. Tinazzi et al. [2005] have shown that prolonged somatosensory stimulation with TENS induces long-lasting depression in MEPs from the target muscle while having the opposite effect (i.e., long-lasting facilitation) on the nontarget muscles, however, this was not confirmed by others [Fernandez-del-Olmo et al., 2008]. Nevertheless, the suggestion of simultaneous changes in excitatory and inhibitory pathways made by Tinazzi et al. [2005] may be one way in which it is possible to account for the simultaneous enlargement of cortical motor representations in the stimulated (APB) and nonstimulated muscles in the absence of a change in global excitability (e.g., rMT) of the descending motor pathways to these muscles. In this respect, the contribution of the GABAergic inhibitory system to the emergence of TENS-induced plasticity may remain a viable but not exclusive means for inducing cortical map changes, as observed in the present study. In the absence of direct recordings of synaptic dynamics or pharmacological manipulation, any inference about the physiological basis of the present findings must be made with caution.

Even though the present findings are encouraging, some limitations need to be recognized. First, no follow-up measurements were conducted beyond the postintervention test to examine the lasting effects of the intervention. Second, no sham TENS group beyond our current control group was included to examine placebo effects. However, as participants were healthy and naïve about the purpose of the study, it is unlikely that our observations were biased by placebo effects. Finally, changes in the motor representation of the hand may be caused by unspecific reasons such as attentional drift away from the zone of stimulation. However, observations from the present and other studies [e.g., Cuypers et al., in press; Ridding et al., 2001] suggest that TENS-induced modulations in cortical motor representations (or tactile sensitivity) extended beyond the boundaries of the stimulated zone, indicating spread of activation from stimulated to nonstimulated parts of the somatosensory network.

\section{Clinical Applications}

The present observations highlight the potential of somatosensory stimulation to serve as a useful complementary therapy in neurorehabilitation. We have shown that TENS-induced enlargements in cortical motor maps were not restricted to the stimulated muscle but also extended to other hand and forearm muscles. However, therapeutic choices for global versus local effects will have to be made according to the specific disorder under treatment. In any case, it is clear that interventions that are first and foremost sensory in nature, do impact upon motor representations that persist for more than $20 \mathrm{~h}$ following the intervention. More research will be necessary to differentially "tune" M1 reorganization by further manipulation of the type of somatosensory stimulation and its parameters.

Since only healthy volunteers were included in this study, generalization of the results to patient populations should be made with caution. However, a recent study using exactly the same intervention protocol (i.e. TENS to the right $\mathrm{APB}$ muscle, $1 \mathrm{~h}$ a day for 3 weeks) in patients with MS, showed long-lasting improvements in tactile sensitivity. MS patients reached the same level of sensitivity as healthy subjects immediately after the intervention, and significant long-term effects were reported up to 3 weeks 
following the last intervention [Cuypers et al., in press]. Overall, this suggests that TENS may be particularly useful as a complementary therapeutic tool in neurorehabilitation.

\section{ACKNOWLEDGMENTS}

The authors wish to thank B. Bories, J. Kissembeeck, B. Kremers, J. Wouters, R. Vanham, K. Govaerts (Rehabilitation and Health Care Research Center, Department of Health Care, University College of Limburg, Hasselt, Belgium) for their crucial assistance in collecting and analyzing the data.

\section{REFERENCES}

Armutlu K, Meric A, Kirdi N, Yakut E, Karabudak R (2003): The effect of transcutaneous electrical nerve stimulation on spasticity in multiple sclerosis patients: A pilot study. Neurorehabil Neural Repair 17:79-82.

Bending J, Cleeves L (1990): Effect of electrical nerve stimulation on dystonic tremor. Lancet 336:1385-1386.

Carel C, Loubinoux I, Boulanouar K, Manelfe C, Rascol O, Celsis P, Chollet F (2000): Neural substrate for the effects of passive training on sensorimotor cortical representation: A study with functional magnetic resonance imaging in healthy subjects. J Cereb Blood Flow Metab 20:478-484.

Caria MA, Kaneko T, Kimura A, Asanuma H (1997): Functional organization of the projection from area 2 to area 4 gamma in the cat. J Neurophysiol 77:3107-3114.

Classen J, Liepert J, Wise SP, Hallett M, Cohen LG (1998): Rapid plasticity of human cortical movement representation induced by practice. J Neurophysiol 79:1117-1123.

Cohen LG, Bandinelli S, Findley TW, Hallett M (1991): Motor reorganization after upper limb amputation in man. A study with focal magnetic stimulation. Brain 114 (Part 1B):615-627.

Conforto AB, Ferreiro KN, Tomasi C, dos Santos RL, Moreira VL, Marie SK, Baltieri SC, Scaff M, Cohen LG (2010): Effects of somatosensory stimulation on motor function after subacute stroke. Neurorehabil Neural Repair 24:263-272.

Cuypers K, Levin O, Thijs H, Swinnen SP, Meesen RLJ: Longterm TENS treatment improves tactile sensitivity in MS patients. Neurorehabil Neural Repair (in press).

Deletis V, Schild JH, Beric A, Dimitrijevic MR (1992): Facilitation of motor evoked potentials by somatosensory afferent stimulation. Electroencephalogr Clin Neurophysiol 85:302-310.

Duclos C, Roll R, Kavounoudias A, Roll JP (2007): Cerebral correlates of the "Kohnstamm phenomenon": An fMRI study. Neuroimage 34:774-783.

Fernandez-del-Olmo M, varez-Sauco M, Koch G, Franca M, Marquez G, Sanchez JA, Acero RM, Rothwell JC (2008): How repeatable are the physiological effects of TENS? Clin Neurophysiol 119:1834-1839.

Foley-Nolan D, Kinirons M, Coughlan RJ, O'Connor P (1990): Post whiplash dystonia well controlled by transcutaneous electrical nervous stimulation (TENS): Case report. J Trauma 30:909-910.

Forner-Cordero A, Steyvers M, Levin O, Alaerts K, Swinnen SP (2008): Changes in corticomotor excitability following prolonged muscle tendon vibration. Behav Brain Res 190:41-49.
Fraser C, Power M, Hamdy S, Rothwell J, Hobday D, Hollander I, Tyrell P, Hobson A, Williams S, Thompson D (2002): Driving plasticity in human adult motor cortex is associated with improved motor function after brain injury. Neuron 34:831-840.

Fuhr P, Cohen LG, Dang N, Findley TW, Haghighi S, Oro J, Hallett M (1992): Physiological analysis of motor reorganization following lower limb amputation. Electroencephalogr Clin Neurophysiol 85:53-60.

Fung J, Barbeau H (1994): Effects of conditioning cutaneomuscular stimulation on the soleus H-reflex in normal and spastic paretic subjects during walking and standing. J Neurophysiol 72:2090-2104.

Ghosh S, Porter R (1988): Corticocortical synaptic influences on morphologically identified pyramidal neurones in the motor cortex of the monkey. J Physiol 400:617-629.

Goulet C, Arsenault AB, Bourbonnais D, Laramee MT, Lepage $Y$ (1996): Effects of transcutaneous electrical nerve stimulation on H-reflex and spinal spasticity. Scand J Rehabil Med 28:169176 .

Hamdy S, Rothwell JC, Aziz Q, Singh KD, Thompson DG (1998): Long-term reorganization of human motor cortex driven by short-term sensory stimulation. Nat Neurosci 1:64-68.

Harms KJ, Rioult-Pedotti MS, Carter DR, Dunaevsky A (2008): Transient spine expansion and learning-induced plasticity in layer 1 primary motor cortex. J Neurosci 28:5686-5690.

Huerta MF, Pons TP (1990): Primary motor cortex receives input from area 3a in macaques. Brain Res 537:367-371.

Kimura I, Ayyar DR, Lippmann SM (1983): Electrophysiological verification of the ulnar to median nerve communications in the hand and forearm. Tohoku J Exp Med 141:269-274.

Krause T, Kurth R, Ruben J, Schwiemann J, Villringer K, Deuchert M, Moosmann M, Brandt S, Wolf K, Curio G, Villringer A (2001): Representational overlap of adjacent fingers in multiple areas of human primary somatosensory cortex depends on electrical stimulus intensity: An fMRI study. Brain Res 899:36-46.

Kurth R, Villringer K, Curio G, Wolf KJ, Krause T, Repenthin J, Schwiemann J, Deuchert M, Villringer A (2000): fMRI shows multiple somatotopic digit representations in human primary somatosensory cortex. Neuroreport 11:1487-1491.

Levin MF, Hui-Chan CW (1992): Relief of hemiparetic spasticity by TENS is associated with improvement in reflex and voluntary motor functions. Electroencephalogr Clin Neurophysiol 85:131-142.

Lewis GN, Byblow WD (2004): The effects of repetitive proprioceptive stimulation on corticomotor representation in intact and hemiplegic individuals. Clin Neurophysiol 115:765-773.

Liepert J, Bauder H, Wolfgang HR, Miltner WH, Taub E, Weiller C (2000): Treatment-induced cortical reorganization after stroke in humans. Stroke 31:1210-1216.

Lucier GE, Ruegg DC, Wiesendanger M (1975): Responses of neurones in motor cortex and in area $3 \mathrm{~A}$ to controlled stretches of forelimb muscles in cebus monkeys. J Physiol 251:833-853.

Mace MJ, Levin O, Alaerts K, Rothwell JC, Swinnen SP (2008): Corticospinal facilitation following prolonged proprioceptive stimulation by means of passive wrist movement. J Clin Neurophysiol 25:202-209.

Matteis M, Vernieri F, Troisi E, Pasqualetti P, Tibuzzi F, Caltagirone C, Silvestrini M (2003): Early cerebral hemodynamic changes during passive movements and motor recovery after stroke. J Neurol 250:810-817. 
Miller L, Mattison P, Paul L, Wood L (2007): The effects of transcutaneous electrical nerve stimulation (TENS) on spasticity in multiple sclerosis. Mult Scler 13:527-533.

Murphy JT, Wong YC, Kwan HC (1975): Afferent-efferent linkages in motor cortex for single forelimb muscles. J Neurophysiol 38:990-1014.

Naito E, Ehrsson HH, Geyer S, Zilles K, Roland PE (1999): Illusory arm movements activate cortical motor areas: A positron emission tomography study. J Neurosci 19:6134-6144.

Nelles G, Spiekramann G, Jueptner M, Leonhardt G, Muller S, Gerhard H, Diener HC (1999): Evolution of functional reorganization in hemiplegic stroke: A serial positron emission tomographic activation study. Ann Neurol 46:901-909.

Nelles G, Jentzen W, Jueptner M, Muller S, Diener HC (2001): Arm training induced brain plasticity in stroke studied with serial positron emission tomography. Neuroimage 13:11461154 .

Ng SS, Hui-Chan CW (2007): Transcutaneous electrical nerve stimulation combined with task-related training improves lower limb functions in subjects with chronic stroke. Stroke 38:2953-2959.

Nudo RJ, Wise BM, SiFuentes F, Milliken GW (1996): Neural substrates for the effects of rehabilitative training on motor recovery after ischemic infarct. Science 272:1791-1794.

Oldfield RC (1971): The assessment and analysis of handedness: The Edinburgh inventory. Neuropsychologia 9:97-113.

Pascual-Leone A, Cammarota A, Wassermann EM, Brasil-Neto JP, Cohen LG, Hallett M (1993): Modulation of motor cortical outputs to the reading hand of braille readers. Ann Neurol 34:33-37.

Pascual-Leone A, Grafman J, Hallett M (1994): Modulation of cortical motor output maps during development of implicit and explicit knowledge. Science 263:1287-1289.

Pascual-Leone A, Nguyet D, Cohen LG, Brasil-Neto JP, Cammarota A, Hallett M (1995): Modulation of muscle responses evoked by transcranial magnetic stimulation during the acquisition of new fine motor skills. J Neurophysiol 74:10371045.

Quartarone A, Bagnato S, Rizzo V, Siebner HR, Dattola V, Scalfari A, Morgante F, Battaglia F, Romano M, Girlanda P (2003): Abnormal associative plasticity of the human motor cortex in writer's cramp. Brain 126:2586-2596.

Quartarone A, Rizzo V, Bagnato S, Morgante F, Sant'Angelo A, Girlanda P, Siebner HR (2006): Rapid-rate paired associative stimulation of the median nerve and motor cortex can produce long-lasting changes in motor cortical excitability in humans. J Physiol 575:657-670.

Radovanovic S, Korotkov A, Ljubisavljevic M, Lyskov E, Thunberg J, Kataeva G, Danko S, Roudas M, Pakhomov S, Medvedev S, Johansson H (2002): Comparison of brain activity during different types of proprioceptive inputs: A positron emission tomography study. Exp Brain Res 143:276-285.

Ridding MC, Rothwell JC (1997): Stimulus/response curves as a method of measuring motor cortical excitability in man. Electroencephalogr Clin Neurophysiol 105:340-344.

Ridding MC, McKay DR, Thompson PD, Miles TS (2001): Changes in corticomotor representations induced by prolonged peripheral nerve stimulation in humans. Clin Neurophysiol 112:1461-1469.

Rioult-Pedotti MS, Donoghue JP, Dunaevsky A (2007): Plasticity of the synaptic modification range. J Neurophysiol 98:36883695 .
Rosenkranz K, Rothwell JC (2003): Differential effect of muscle vibration on intracortical inhibitory circuits in humans. J Physiol 551:649-660.

Rosenkranz K, Rothwell JC (2006): Differences between the effects of three plasticity inducing protocols on the organization of the human motor cortex. Eur J Neurosci 23:822-829.

Rosenkranz K, Kacar A, Rothwell JC (2007): Differential modulation of motor cortical plasticity and excitability in early and late phases of human motor learning. J Neurosci 27:12058-12066.

Rosenkranz K, Butler K, Williamon A, Cordivari C, Lees AJ, Rothwell JC (2008): Sensorimotor reorganization by proprioceptive training in musician's dystonia and writer's cramp. Neurology 70:304-315.

Roth BJ, Saypol JM, Hallett M, Cohen LG (1991): A theoretical calculation of the electric field induced in the cortex during magnetic stimulation. Electroencephalogr Clin Neurophysiol 81:47-56.

Sato K, Nariai T, Tanaka Y, Maehara T, Miyakawa N, Sasaki S, Momose-Sato Y, Ohno K (2005): Functional representation of the finger and face in the human somatosensory cortex: intraoperative intrinsic optical imaging. Neuroimage 25:12921301.

Siebner HR, Rothwell J (2003): Transcranial magnetic stimulation: new insights into representational cortical plasticity. Exp Brain Res 148:1-16.

Skeil D, Thorpe AC (2001): Transcutaneous electrical nerve stimulation in the treatment of neurological patients with urinary symptoms. BJU Int 88:899-908.

Sonde L, Gip C, Fernaeus SE, Nilsson CG, Viitanen M (1998): Stimulation with low frequency $(1.7 \mathrm{~Hz})$ transcutaneous electric nerve stimulation (low-tens) increases motor function of the post-stroke paretic arm. Scand J Rehabil Med 30:95-99.

Steyvers M, Levin O, Van Baelen M, Swinnen SP (2003a): Corticospinal excitability changes following prolonged muscle tendon vibration. Neuroreport 14:1901-1905.

Steyvers M, Levin O, Verschueren SM, Swinnen SP (2003b): Frequency-dependent effects of muscle tendon vibration on corticospinal excitability: A TMS study. Exp Brain Res 151:914.

Thickbroom GW, Sammut R, Mastaglia FL (1998): Magnetic stimulation mapping of motor cortex: Factors contributing to map area. Electroencephalogr Clin Neurophysiol 109:79-84.

Thickbroom GW, Byrnes ML, Mastaglia FL (1999): Methodology and application of TMS mapping. Electroencephalogr Clin Neurophysiol Suppl 51:48-54.

Tinazzi M, Zarattini S, Valeriani M, Romito S, Farina S, Moretto G, Smania N, Fiaschi A, Abbruzzese G (2005): Long-lasting modulation of human motor cortex following prolonged transcutaneous electrical nerve stimulation (TENS) of forearm muscles: Evidence of reciprocal inhibition and facilitation. Exp Brain Res 161:457-464.

Tinazzi M, Zarattini S, Valeriani M, Stanzani C, Moretto G, Smania N, Fiaschi A, Abbruzzese G (2006): Effects of transcutaneous electrical nerve stimulation on motor cortex excitability in writer's cramp: Neurophysiological and clinical correlations. Mov Disord 21:1908-1913.

Toglia JU, Izzo K (1985): Treatment of myoclonic dystonia with transcutaneous electrical nerve stimulation. Ital J Neurol Sci 6:75-78.

Wassermann EM (1998): Risk and safety of repetitive transcranial magnetic stimulation: Report and suggested guidelines from the international workshop on the safety of repetitive 
- Meesen et al.

transcranial magnetic stimulation, June 5-7, 1996. Electroencephalogr Clin Neurophysiol 108:1-16.

Wassermann EM, McShane LM, Hallett M, Cohen LG (1992): Noninvasive mapping of muscle representations in human motor cortex. Electroencephalogr Clin Neurophysiol $85: 1-8$.

Wilson SA, Thickbroom GW, Mastaglia FL (1993): Transcranial magnetic stimulation mapping of the motor cortex in normal subjects. The representation of two intrinsic hand muscles. J Neurol Sci 118:134-144.
Wilson SA, Day BL, Thickbroom GW, Mastaglia FL (1996): Spatial differences in the sites of direct and indirect activation of corticospinal neurones by magnetic stimulation. Electroencephalogr Clin Neurophysiol 101:255-261.

Zarzecki P, Shinoda Y, Asanuma H (1978): Projection from area $3 a$ to the motor cortex by neurons activated from group I muscle afferents. Exp Brain Res 33:269-282.

Ziemann U, Rothwell JC, Ridding MC (1996): Interaction between intracortical inhibition and facilitation in human motor cortex. J Physiol 496 (Part 3):873-881. 